\title{
Condições visuais autorrelatadas e quedas em idosos institucionalizados
}

\section{Self-reported visual conditions and falls in institutionalized elderly}

Ruth Losada de Menezes', Maria Márcia Bachion²

\section{ResUmo}

Objetivo: Avaliar as condições visuais autorrelatadas por idosos institucionalizados e sua relação com acidentes por quedas. Métodos: Estudo prospectivo em que foram entrevistados 59 idosos residentes em instituições de longa permanência para idosos, em Goiânia, GO, entre 2005 e 2007. Foram realizados dois inquéritos com intervalo de dois anos para realização das avaliações propostas. As condições visuais no primeiro inquérito e o relato de quedas no seguimento foram autorrelatadas. Os idosos foram classificados segundo a presença de déficit visual e comparados quanto à ocorrência de queda com o teste Exato de Fisher. Resultados: Déficit visual foi relatado por 48 $(81,4 \%)$ dos idosos e os acidentes por quedas atingiram 32 (54,2\%) dos mesmos, sendo estes mais prevalentes entre idosos com déficit visual. Após o primeiro inquérito, no seguimento de dois anos, não foi encontrada diferença estatisticamente significativa $(\mathrm{p}=0,171)$ entre a variável déficit visual autorrelatado e quedas. Conclusão: Os dados encontrados sinalizam a necessidade de melhor investigação clínica oftalmológica dos idosos. O diagnóstico precoce de comprometimentos visuais e/ou a correção dos mesmos pode beneficiar os idosos e evitar prejuízos funcionais futuros advindos destes e de consequências como as quedas.

Descritores: Visão; Acidentes por quedas; Envelhecimento; Idoso; Saúde do idoso institucionalizado

\section{Abstract}

Objective: To evaluate the visual conditions self-reported by institutionalized elderly and their relation to accidental falls. Methods: Prospective study in which we interviewed 59 elderly residents of long-stay institutions for elderly in Goiânia, between 2005 and 2007. Two surveys were conducted with an interval of two years to perform the assessments proposed. The visual conditions in the first survey and the report of falls in the wake were self-reported. The elderly were classified according to the presence of visual impairment and compared the occurrence of fall with the Fisher exact test. Results: Visual deficit was reported by 48 (81.4\%) of the elderly and accidents from falls reached $32(54.2 \%)$ of them; which were more prevalent among older adults with visual impairment. After the first inquiry, in the following two years, found no statistically significant difference $(\mathrm{p}=0.171)$ between the variable selfreported visual impairment and falls. Conclusion: The findings indicate the need for better research ophthalmology clinic for the elderly. Early diagnosis of visual impairment and / or correct it can benefit the elderly and to avoid future functional impairment arising from these and consequences such as falls.

Keywords: Vision; Accidental falls; Aging; Aged; Health of institutionalized elderly

\footnotetext{
'Universidade Federal de Goiás (UFG) - Goiânia (GO), Brasil; Universidade de Brasília (UnB) Campus UnB Ceilândia - Brasília (DF), Brasil; ${ }^{2}$ Faculdade de Enfermagem da Universidade Federal de Goiás - UFG - Goiânia (GO), Brasil.

Trabalho realizado na Faculdade de Medicina, Programa de Pós-graduação em Ciências da Saúde da Universidade Federal de Goiás (UFG) - Goiânia (GO), Brasil

Os autores declaram inexistir conflitos de interesse
}

Recebido para publicação em 17/1/2011 - Aceito para publicação em 29/3/2011 


\section{INTRODUÇÃO}

A longevidade é uma realidade e muitas vezes é acompanhada de perdas que vão ocorrendo ao longo da vida, culminando no processo de institucionalização. O idoso institucionalizado, no Brasil, apresenta características peculiares, pois além de apresentar alterações fisiológicas e patológicas que podem ocorrer no processo de envelhecimento, e que acontecem independentemente do ambiente em que se vive, é marcado por sentimentos de solidão e exclusão familiar, sendo estes alguns dos fatores determinantes para o seu ingresso na instituição de longa permanência. Este cenário requer uma mudança de prioridades com oferta de serviços relacionados à saúde e suporte a esta população.

Um dos primeiros sistemas a sofrer o impacto do envelhecimento fisiológico é o sistema sensorial e, particularmente, o visual. Idosos com baixa acuidade visual tendem a apresentar déficit de controle postural, comprometimento funcional e maior risco de quedas ${ }^{(1,2)}$. A catarata é uma das doenças oculares de maior prevalência em idosos, levando a uma diminuição na acuidade visual, na sensibilidade ao contraste e na percepção de cor. Quando associada a pouca iluminação ambiental, favorece escorregões e derrapadas em superfícies irregulares do chão do ambiente (ex.: poças, pontas de tapetes erguidas, batentes de portas) $)^{(3)}$.

Vários estudos apontam para uma relação estreita entre déficits visuais, quedas e fraturas de quadril em $\operatorname{idosos}^{(4-7)}$. Condições como a catarata e degeneração macular relacionada à idade estão presentes em pacientes admitidos em enfermarias geriátricas devido às quedas $^{(5)}$. Estudos nacionais ${ }^{(8-11)}$ também têm evidenciado a associação das quedas com déficits visuais em idosos; fatores de risco como pobre acuidade visual, sensibilidade de contraste reduzida e diminuição do campo visual têm sido associados à ocorrência de duas ou mais quedas relatadas por idosos no ano anterior ${ }^{(12)}$.

O conhecimento das alterações relacionadas à função visual durante o processo de envelhecimento e as principais patologias visuais que afetam esta faixa etária, faz com que a população assuma seu papel na prevenção e na busca de um envelhecimento bem-sucedido, assim como direciona a comunidade oftalmológica para a gerontologia, considerando que o envelhecimento populacional é fato ${ }^{(13)}$. Vários dos problemas relacionados à baixa visão em idosos são passíveis de tratamento e correção, por meio de órteses ou mesmo extração da cata$\operatorname{rata}^{(14)}$, melhorando, desta forma, a função visual.

O objetivo deste estudo é avaliar as condições visuais autorrelatadas por idosos institucionalizados e sua relação com acidentes por quedas.

\section{Métodos}

Os dados da pesquisa foram obtidos no primeiro e segundo inquérito de um estudo maior denominado "Quedas e fatores multidimensionais associados: estudo longitudinal de idosos residentes em instituições de longa permanência em Goiânia (GO)" (15), entre os anos de 2005 e 2007. A amostra do estudo foi selecionada por busca sistemática em cinco instituições filantrópicas de longa permanência para idosos na cidade de Goiânia, Goiás. Todas as cinco instituições foram visitadas, e os 250 idosos institucionalizados foram convidados a participar do estudo. Desses, 93 não preencheram os critérios de inclusão ${ }^{(16)}, 7$ recusaram-se e 55 não participaram da pesquisa porque estavam física ou cognitivamente incapacitados para a realização das avaliações propostas, desta forma, participaram do primeiro inquérito (2005) do estudo 95 idosos. No ano de 2007, a partir da amostra inicial de 95 idosos, sendo destes 52,7\% mulheres $(\mathrm{n}=50)$ e $47,3 \%$ homens $(n=45)$, foram excluídos do segundo inquérito 36 idosos, uma vez que, nesse seguimento de dois anos (2005-2007), 13 foram a óbito, 02 apresentaram déficit cognitivo, 14 retornaram para o convívio familiar, 05 não aceitaram participar do segundo inquérito e 2 não foram encontrados na instituição. Dessa forma, foram investigados para o presente estudo 59 idosos que participaram dos dois inquéritos.

A amostra total foi composta por $57,6 \%(n=34)$ de mulheres e $42,4 \%$ de homens $(n=25)$. A média de idade foi de 78,4 $\pm 7,8$ anos. Mais da metade dos idosos investigados $(69,3 \%)$ apresentavam tempo de institucionalização de um a dez anos. No que se refere ao estado civil, prevaleceu situação "atualmente solteiro" (61\%), destacando a predominância de pessoas viúvas. $49,5 \%$ da amostra eram analfabetos, $42,3 \%$ tinham ensino fundamental e $8,6 \%$ possuíam ensino médio/superior.

Foram coletadas informações autorrelatadas a respeito das condições visuais e ocorrência de queda. A autoavaliação devido à facilidade com que pode ser obtida tem sido bastante utilizada como indicador de saúde em diversos estudos epidemiológicos ${ }^{(17-18)}$. Os episódios de queda considerados foram os ocorridos no seguimento de maio/agosto de 2005 a maio/agosto de 2007, e para a investigação de sua relação com as condições visuais, estas foram as avaliadas no primeiro inquérito. A definição de queda considerada neste estudo foi de qualquer incidente no qual o paciente venha ao chão ou a um nível 
inferior independentemente de sua vontade.

Foi realizada análise descritiva das variáveis de interesse. Para correlacionar o evento queda relatado no segundo inquérito com as condições visuais autorrelatadas no primeiro inquérito foi foram empregados a frequência simples e o teste exato de Fisher. O nível de significância adotado para os testes estatísticos foi de $5 \%$.

O estudo teve aprovação do Comitê de Ética em Pesquisa da Universidade Federal de Goiás (Protocolos CEP 032/2004 e 045/2007).

\section{Resultados}

Déficit visual foi relatado por 48 (81,4\%) dos idosos. Destes, $26(44,1 \%)$ apresentaram déficit corrigido com órtese e 22 (37,3\%) déficit não corrigido (Tabela 1). No seguimento de dois anos a partir do primeiro inquérito realizado no ano de 2005, dos 59 idosos investigados, $32(54,2 \%)$ relataram ter sofrido queda, 22 $(68,8 \%)$ idosos sofreram um episódio, $06(18,8 \%)$ dois e $04(12,5 \%)$ três.

Ao buscar associação entre a variável déficit visual autorrelatado e quedas ocorridas após o primeiro inquérito, no seguimento de dois anos, não foi encontrada diferença estatisticamente significativa $(p=0,171)$ (Figura 1).

\section{DiscussÃo}

O sistema visual é um importante contribuinte para o equilíbrio, fornecendo informações sobre o ambiente e a localização, a direção e a velocidade de movimento do indivíduo ${ }^{(19)}$. No entanto, para ocorrer o controle postural são necessárias outras aferências sensoriais provenientes dos sistemas vestibular e somatossensorial. Apesar de cada um dos sistemas fornecerem diferentes tipos de informação para o sistema de controle postural, a ação individual de cada um deles não é suficiente para obter informações acuradas da posição do centro de gravidade corporal no espaço. Todas as informações provenientes destes sistemas devem ser integradas ao sistema de controle postural a fim de proporcionar uma melhor representação da posição e dos movimentos do centro de gravidade e, deste modo, proporcionar um controle postural efetivo e flexível ${ }^{(20-}$ ${ }^{21}$. A privação ou alteração em qualquer um destes sistemas pode predispor o idoso ao déficit de equilíbrio, à dependência funcional e a episódios de quedas.

A catarata, as alterações na retina e no nervo óptico e o glaucoma são as principais causas do compro-
Tabela 1

Condições visuais autorrelatadas por idosos institucionalizados, em Goiânia (GO), 2005-2007

\begin{tabular}{lcr}
\hline Condições visuais autorrelatadas & N & \% \\
\hline Déficits visuais & 48 & 81,4 \\
Déficit corrigido com órtese & 26 & 44,1 \\
Déficit não corrigido & 22 & 37,3 \\
Sem déficits visuais & 11 & 18,6 \\
Total & 59 & 100 \\
\hline
\end{tabular}

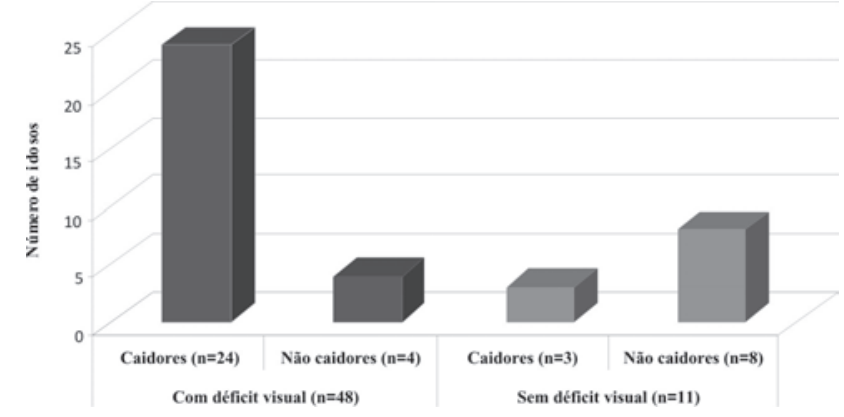

Figura 1: Distribuição das variáveis relacionadas com condições visuais auto-relatadas por idosos institucionalizados e ocorrência de quedas, em Goiânia (Goiás), 2005-2007

metimento visual entre os idosos. A maior parte das alterações visuais decorrentes do envelhecimento é inevitável, no entanto pode receber uma abordagem preventiva, corretiva e curativa, sendo, pelo menos, $\operatorname{adiadas}^{(22)}$.

Os déficits visuais levam a menor comunicação visual, dependência e restrição de mobilidade, inclusive levando à maior probabilidade de traumas em geral. Os distúrbios visuais, ao desencadearem maior dependência física, podem gerar também dificuldades nos aspectos psicoemocionais para os idosos ${ }^{(23)}$.

As quedas são eventos adversos de origem multifatorial e resultantes de uma interação entre fatores intrínsecos e ambientais, sendo que o risco de sofrer um acidente como este é maior para pessoas com limitações visuais quando comparado com a população em geral( ${ }^{(24)}$.

Existem evidências de que há esforço considerável de coordenação entre os movimentos dos olhos e os movimentos dos membros inferiores durante a locomoção e com o envelhecimento, o tempo para processamento da informação visual é prolongado para obter sucesso durante a deambulação ${ }^{(25)}$. O comprometimento visual aumenta o risco de tropeçar em obstáculos ambientais e / ou perder equilíbrio em diferentes 
situações $^{(26)}$, causando, ainda, incerteza durante a locomoção e adoção de marcha insegura devido à incompleta ou pouco confiável informação visual sobre a localização de objetos no ambiente. Além disso, o risco de queda é agravado em certos grupos, como os idosos, que tendem a ser mais dependentes da visão para a manutenção da postura ortostática ${ }^{(27)}$.

Embora a literatura indique associação entre déficits visuais ${ }^{(4,5,6-11,24,27-28)} \mathrm{com}$ a queda, isto não foi observado no presente estudo. Fatores de risco incluem a redução da acuidade visual ${ }^{(24,29)}$, a redução da sensibilidade de contraste ${ }^{(24,30)}$, déficit visual autorrelatado ${ }^{(8-1)} \mathrm{e}$ diminuição do campo visual ${ }^{(12,24)}$.

Intervenções oftálmicas podem ser particularmente importantes para idosos residentes em instituições de longa permanência onde existe elevada prevalência de deficiência visual e uma elevada incidência de queda ${ }^{(31)}$.

\section{Conclusão}

Com este estudo foi possível verificar elevada ocorrência de déficit visual autorrelatado pelos idosos, sendo que os acidentes por queda foram mais prevalentes justamente neste grupo. É sabido que as intervenções para a prevenção de quedas são multidimensionais, no entanto, chamamos a atenção para o componente visual destes idosos investigados. Embora os dados deste estudo não permitam ser extrapolados para a população em geral, visto tratar-se de uma amostra relativamente pequena, a indentificação, nesta pesquisa, de percentual elevado de idosos acidentados por quedas com a baixa da acuidade visual relacionam esses dois fatores como relevantes na incapacitação das pessoas nesta faixa etária. $\mathrm{O}$ diagnóstico precoce de comprometimentos visuais e/ou a correção dos mesmos pode beneficiar significativamente os idosos e evitar prejuízos funcionais futuros advindos destes e de consequências como as quedas.

\section{REFERÊNCIAS}

1. Macedo BG, Pereira LSM, Gomes PF, Silva JP, Castro ANV. Impacto das alterações visuais nas quedas, desempenho funcional, controle postural e no equilíbrio dos idosos: uma revisão de literatura. Rev Bras Geriatr Gerontol. 2008;11(3):419-32.

2. Lamoureux E, Gadgil S, Pesudovs K, Keeffe J, Fenwick E, Dirani M, et al. The relationship between visual function, duration and main causes of vision loss and falls in older people with low vision. Graefes Arch Clin Exp Ophthalmol. 2010;248(4):527-33.
3. Tideiksaar R. As quedas na velhice: prevenção e cuidados. 2a ed. São Paulo: Organização Andrei Editora; 2003.

4. Lord SR, Dayhew J. Visual risk factors for falls in older people. J Am Geriatr Soc. 2001;49(5):508-15. Comment in: J Am Geriatr Soc. 2001;49(5):676-7.

5. Abdelhafiz AH, Austin CA. Visual factors should be assessed in older people presenting with falls or hip fracture. Age Ageing. 2003;32(1):26-30. Review.

6. Klein BE, Moss SE, Klein R, Lee KE, Cruickshanks KJ. Associations of visual function with physical outcomes and limitations 5 years later in an older population: the Beaver Dam eye study. Ophthalmology. 2003;110(4):644-50.

7. Lord SR. Visual risk factors for falls in older people. Age Ageing. 2006;35 Suppl 2:ii42-ii45. Review.

8. Perracini MP, Ramos LR. Fatores associados a quedas em uma coorte de idosos residentes na comunidade. Rev Saúde Pública. 2002;36(6):709-16.

9. Santos MLC, Andrade MC. Incidência de quedas relacionada aos fatores de risco em idosos institucionalizados. Rev Baiana Saúde Pública. 2005;29(1):57-68.

10. Menezes RL, Bachion MM. Estudo da presença de fatores de risco intrínsecos para quedas, em idosos institucionalizados. Ciênc Saúde Coletiva. 2008;13(4):1209-18.

11. Siqueira FC. Quedas e fatores associados em idosos institucionalizados no município de Pelotas/RS. Ciênc Saúde Coletiva [Internet]. 2010 mar.; [cerca de 15 p.] [citado 2010 Dez 17]. Disponível em: http://www.cienciaesaudecoletiva.com.br/ artigos/artigo_int.php?id_artigo=3198 .

12. Ivers RQ, Cumming RG, Mitchell P, Attebo K. Visual impairment and falls in older adults: the Blue Mountains Eye Study. J Am Geriatr Soc. 1998;46(1):58-64.

13. Chaves C. Senescência ocular e o século XXI. Rev Bras Oftalmol. 2010;69(4):215-6.

14. Desapriya E, Subzwari S, Scime-Beltrano G, Samayawardhena LA, Pike I. Vision improvement and reduction in falls after expedited cataract surgery Systematic review and metaanalysis. J Cataract Refract Surg. 2010;36(1):13-9.

15. Menezes RL. Quedas e fatores multidimensionais associados: estudo longitudinal de idosos residentes em instituições de longa permanência em Goiânia (GO). [tese]. Goiânia: Faculdade de Medicina da Universidade Federal de Goiás; 2009.

16. Menezes RL. Quedas em idosos institucionalizados no município de Goiânia. [dissertação]. Goiânia: Faculdade de Medicina da Universidade Federal de Goiás; 2005.

17. Lima-Costa MF, Firmo JOA, Uchôa E. A estrutura da autoavaliação da saúde entre idosos: projeto Bambuí. Rev Saúde Pública. 2004;38(6):827-34.

18. Santos SM, Chor D, Werneck GL, Coutinho ESF. Associação entre fatores contextuais e auto-avaliação de saúde: uma revisão sistemática de estudos multinível. Cad Saúde Pública. 2007;23(11):2533-54.

19. Spirduso WW. Dimensões físicas do envelhecimento. Barueri: Manole; 2005.

20. Freitas Júnior P, Barela JA. Alterações no funcionamento do sistema de controle postural de idosos: Uso da informação visual. Rev Port Ciênc Desp. 2006;6(1):94-105.

21. Kulmala J, Viljanen A, Sipilä S, Pajala S, Pärssinen O, Kauppinen M, et al. Poor vision accompanied with other sensory impairments as a predictor of falls in older women. Age Ageing. 2009;38(2):162-7.

22. Xavier FM, Ferraz MP, Marc N, Escosteguy NU, Moriguchi EH. Elderly people's definition of quality of life. Rev Bras Psiquiatr. 2003;25(1):31-9. 
23. West S, Sommer A. Prevention of blindness and priorities for the future. Bull World Health Organ. 2001;79(3):244-8. Comment in: Bull World Health Organ. 2002;80(5):419.

24. Klein BE, Klein R, Lee KE, Cruickshanks KJ. Performancebased and self-assessed measures of visual function as related to history of falls, hip fractures, and measured gait time. The Beaver Dam Eye Study. Ophthalmology. 1998;105(1):160-4.

25. Chapman GJ, Hollands MA. Age-related differences in visual sampling requirements during adaptive locomotion. Exp Brain Res. 2010;201(3):467-78.

26. Lord SR, Dayhew J, Howland A. Multifocal glasses impair edge-contrast sensitivity and depth perception and increase the risk of falls in older people. J Am Geriatr Soc. 2002;50(11):1760-6.

27. Tobis JS, Block M, Steinhaus-Donham C, Reinsch S, Tamaru K, Weil D. Falling among the sensorially impaired elderly. Arch Phys Med Rehabil. 1990;71(2):144-7.

28. Tromp AM, Pluijm SM, Smit JH, Deeg DJ, Bouter LM, Lips P. Fall-risk screening test: a prospective study on predictors for falls in community-dwelling elderly. J Clin Epidemiol. 2001;54(8):837-44.

29. Tromp E. [Risk profiles and preventive measures of falls in elderly persons]. Tijdschr Gerontol Geriatr. 2002;33(1):21-5. Dutch.

30. Lord SR, Clark RD, Webster IW. Visual acuity and contrast sensitivity in relation to falls in an elderly population. Age Ageing. 1991;20(3):175-81.

31. Black A, Wood J. Vision and falls. Clin Exp Optom. 2005;88(4):212-22. Review.

\section{Endereço para correspondência:}

Ruth Losada de Menezes

Campus UnB Ceilândia, QNN 14 Área Especial,

Guariroba - Ceilândia Sul

CEP 72220-140 - Brasília, (DF), Brasil

Fax: (61) 33766042

E-mail: ruthlosada@unb.br 